\title{
Design Optimization of a High-Power Transformer for Three- Phase Dual Active Bridge DC-DC Converter for MVDC Grids
}

\author{
Youngsil Lee*, Gaurang Vakil*, Ralph Feldman*, Andrew Goodman*, Patrick Wheeler* \\ ^PEMC Group, University Of Nottingham, NG7 2RD, UK. eexyl30@nottingham.ac.uk
}

Keywords: DC-DC converter, 3DAB, transformer design, non-sinusoidal excitation, finite element analysis.

\begin{abstract}
High-power DC-DC converter will be one of the essential technologies for the future DC grids. Especially, a three-phase dual-active bridge DC-DC (3DAB) Converter is highly suitable for high-power DC systems. Key component within this converter is the high power transformer operated at a medium frequency (MF) range. The design and optimization of this key component is presented in this paper. The transformer provides galvanic isolation from low voltage level to medium level or high voltage level and provides stepping up or down of the output (secondary) voltage. At first, a design of three-phase medium frequency transformer is developed. The designed transformer is then validated using two-dimensional (2D) transient finite element analysis (FEA) and performance under the non-sinusoidal excitation is determined. After that, the optimization is carried out, for the set of selected design variables, in order to enhance the power density and efficiency of the targeted transformer. Finally, the performance of the full system, $3 \mathrm{DAB}$ converter, is determined using the parameters of optimized transformer. Also, two different rated input voltages for $4 \mathrm{MW} / 1 \mathrm{kHz}$ threephase transformer are considered and analysed in this paper.
\end{abstract}

\section{Introduction}

DC grids for offshore wind energy have been receiving special research attention. Most important advantages of DC grids for offshore wind farm are low power losses and small size cable for carrying large amounts of power over long distances $[1,2]$. Using 3DAB conversion system, furthermore, a bulky $50 / 60 \mathrm{~Hz}$ transformer can be replaced by MF transformer operating at the kilo-hertz range, resulting in considerable reduction in transformer weight/size. Two possible configurations, one with $V_{l}=690 \mathrm{~V}$ and another with $V_{l}=3.3 \mathrm{kV}$, are considered for the DC grid for offshore wind farm and is shown in Fig. 1. Considering the maximum voltage obtainable from space vector modulation scheme for the maximum modulation index of 0.9 , the maximum $V_{2}$ 's for the respective $V_{l}$ 's are $1.1 \mathrm{kV}$ and $5.2 \mathrm{kV}$. The DC grid include, a PWM rectifier that converts the output voltage of wind turbine into Low-Voltage (LV) DC, a Medium-Voltage (MV) DC-DC converter located in the wind turbine platform that boost's the LV DC to MV DC and an HV DC-DC platform that again boost's up the MV DC to HVDC transmission level. Additional wind turbines can be connected to the MVDC grid for power collection.

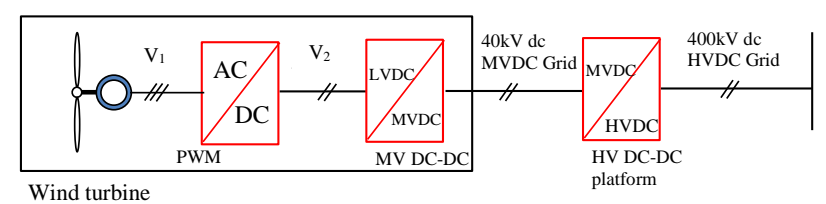

Fig. 1 DC grid for offshore wind farm

In this work, a 4MW wind turbine (WT) system is considered to analyse and validate the proposed systems. Most generators are developed for rated voltage of $690 \mathrm{~V}$ but to improve the efficiency at higher power levels, an alternative solution for rated voltage of $3.3 \mathrm{kV}$ is considered. $\mathrm{ABB}$ [3] and GE [4] have recently developed high power generators for rated voltage of $3.3 \mathrm{kV}$.

$3 \mathrm{DAB}$ Converter which is suitable for high-power application has been studied in $[5,6]$. The 3DAB Converter offers a higher power capability and smaller passive filtering parts compared to single-phase dual active bridge (1DAB). One key component of 3DAB Converter is a high-power galvanic transformer enabling isolation with MV line for a safety operation and also enabling a soft-switching control which can reduces the switching losses. Design of conventional transformers operating at $50 / 60 \mathrm{~Hz}$ has been presented in [710]. For high power application, transformers operating at medium or high frequencies are considered because increase in operating frequency provides significant reduction in the transformer's size and weight leading to reduced manufacturing cost and overall volume of the system. Design of medium/high frequency transformer is presented in [1116], however, they only consider the design procedure of single-phase transformer. More recently, design of threephase transformer for $3 \mathrm{DAB}$ converter topology with the focus on losses has been developed $[6,16]$. The work presented here not only considers power loss analysis but also carries out optimization, for maximizing the efficiency, of three-phase MF transformer operating at $1 \mathrm{kHz}$. Moreover, comparison between the two voltage levels, $690 \mathrm{~V}$ and $3.3 \mathrm{kV}$, is carried out to determine which is the optimal configuration for the MVDC grid.

\section{3DAB Converter}

A 3DAB converter topology is depicted in Fig. 2. The 3DAB Converter contains two active voltage source bridges that are connected to a MF transformer which is connected in Y-Y 
type to reduce the circulating current and reduce the unbalanced currents. The primary side bridge converts the dc input voltage into the MF ac voltage that is applied the MF transformer and then the voltage at the secondary terminals of the transformer is rectified by the secondary side bridge.

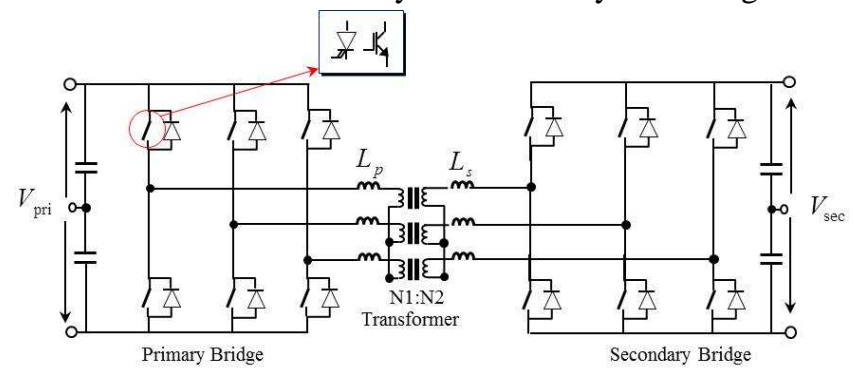

Fig. 2 A 3DAB converter topology

The MF transformer is providing the galvanic isolation. The average output power $\left(P_{o}\right)$ can be calculated as

$$
\begin{gathered}
P_{o}=\frac{V_{i n}^{2}}{\omega_{s w} L_{\sigma}} \cdot d \cdot \varphi\left[\frac{2}{3}-\frac{\varphi}{2 \pi}\right] \quad \text { for }(0 \leq \varphi \leq \pi / 3) \\
P_{o}=\frac{V_{i n}^{2}}{\omega_{s w} L_{\sigma}} d\left[\varphi-\frac{\varphi^{2}}{\pi}-\frac{\pi}{18}\right] \quad \text { for }(\pi / 3 \leq \varphi \leq 2 \pi / 3)
\end{gathered}
$$

The transformer size and reactive power are determined by apparent power of Y-Y transformer which can be calculated as

$$
S_{T}=3 \cdot V_{p n} \cdot I_{p r m s}
$$

RMS values of phase current at primary side of the transformer can be computed from the following

$$
\begin{gathered}
I_{p r m s}=\frac{V_{i n}}{6 \pi \cdot f_{s w} \cdot L_{\sigma}} \cdot \varphi \cdot \sqrt{\frac{2 \pi-\varphi}{\pi}} \text { for }(0 \leq \varphi \leq \pi / 3) \\
I_{p r m s}=\frac{V_{i n}}{12 \pi \cdot f_{s w} \cdot L_{\sigma}} \cdot \varphi \cdot \sqrt{\frac{8 \pi-6 \varphi}{\pi}} \text { for }(\pi / 3 \leq \varphi \leq 2 \pi / 3)
\end{gathered}
$$

Substituting Equation (4), Equation (5) and $V_{p n}=\frac{\sqrt{2}}{3} V_{i n}$ into Equation (3), the apparent power of the transformer can be calculated as

$$
\begin{gathered}
S_{T}=3 \cdot V_{p n} \cdot I_{p r m s}=\frac{\sqrt{2} \cdot V_{i n}^{2}}{6 \pi \cdot f_{s w} \cdot L_{\sigma}} \cdot \varphi \cdot \sqrt{\frac{2 \pi-\varphi}{\pi}} \text { for }(0 \leq \varphi \leq \pi / 3) \\
S_{T}=\frac{\sqrt{2} \cdot V_{i n}^{2}}{12 \pi \cdot f_{s w} \cdot L_{\sigma}} \cdot \varphi \cdot \sqrt{\frac{8 \pi-6 \varphi}{\pi}} \text { for }(\pi / 3 \leq \varphi \leq 2 \pi / 3)
\end{gathered}
$$

\section{Transformer Design and Optimization}

The proposed design procedure for the $3 \mathrm{DAB}$ MF transformer is shown in Fig. 3. In the pre-design phase, the 3DAB converter system is analysed and the basic specifications (turns ratio, non-sinusoidal converter current waveform, apparent power, expected efficiency and $B_{m}$ ) for the design of transformer are determined.

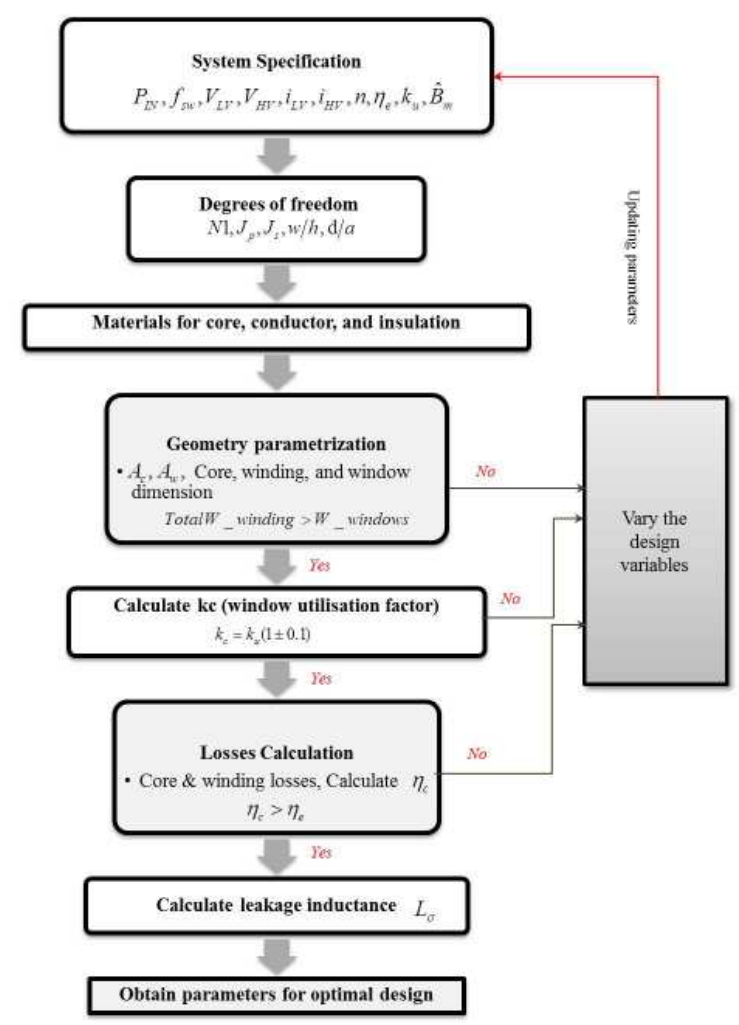

Fig. 3 Design flow-chart of the 3DAB transformer

\subsection{Design of Core}

Using the peak flux density equation in [8], the core cross sectional area, $A_{c}$ can be calculated as

$$
A_{c}=\frac{V_{a n}^{2}}{k_{f} \cdot f_{s w} \cdot \hat{B}_{m} \cdot N_{1}}
$$

Where, $k_{f}$ is waveform coefficient which equal to 4.0 for square waveform. Considering the assumed value for $d / a$, the values of $a$ and $d$ can be calculated from Equation 8 .

The diagram of the three-phase transformer along with the cross sectional view of the limb and with detailed dimension is shown in Fig. 4.
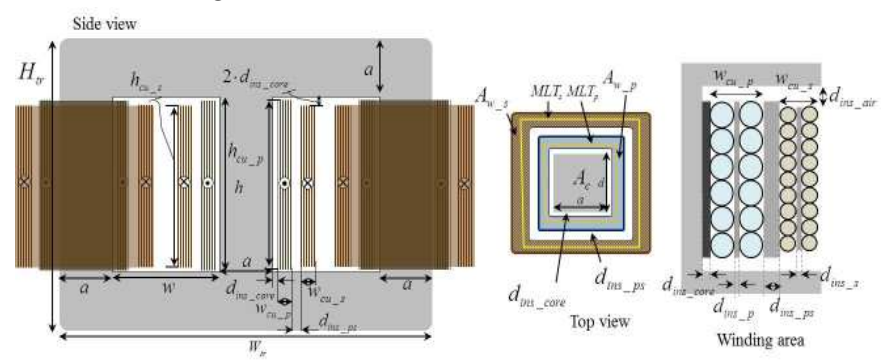

Fig. 4 Detailed dimensions of three-phase transformer

\subsection{Design of Window}

From current density for the given cooling system, the primary and secondary conductor cross section areas can be calculated. In order to keep the skin effect to the minimum, the conductor diameter is kept smaller than twice the skin 
depth of the operating frequency. If the required diameter is larger than twice the skin depth, parallel strands are added to satisfy the condition. Knowing primary and secondary conductor areas and number of primary and secondary turns, the total primary and secondary copper areas can be determined. Total copper area $\left(A_{w_{-} c u}\right)$ is the sum of primary and secondary copper areas. Initially, the value of window utilization factor $k_{u}$ is assumed and the window area $\left(A_{a}\right)$ is calculated as

$$
A_{a}=2 \cdot \frac{A_{w_{-} c u}}{k_{u}}
$$

Again, considering the assumed value for $w / h$, the values of $w$ and $h$ can be calculated from Equation 9 .

Using the core cross section area and the window dimensions, the total core volume ( ool $_{\text {core }}$ in $\mathrm{m}^{3}$ ) can be determined as follows

$$
\text { vol }_{\text {core }}=\left(A_{c} \cdot(3 \cdot h+4 \cdot w)+6 \cdot a^{2} \cdot d\right) \cdot 10^{-9}
$$

The insulation level must meet with international standards. Partial discharge (PD) has to be considered at medium voltage level above $3 \mathrm{kV}$. The insulation design is carried out using DUPONT ${ }^{\mathrm{TM}}$ NOMEX PAPER TYPE insulation with continuous dielectric stress level not exceeding $1.6 \mathrm{kV} / \mathrm{mm}$. A safety factor of 5 is used in the insulation design. Insulation thickness for turns, between layers, between windings and between winding and core is calculated depending upon the potential difference between them. Using this, total insulation cross section area $\left(A_{w_{-} i n s}\right)$ can be calculated. A new value of $k_{u}$, with $10 \%$ increased for safety, is calculated using the Equation 11.

$$
k_{u}=\frac{A_{w_{-} c u}}{1.1 \cdot\left(A_{w_{-} c u}+A_{w_{-} i n s}\right)}
$$

The calculated value of $k_{u}$ should be greater than the assumed value, if not, the calculated value is taken as the initial value and design is repeated. If the no value of $k_{u}$ (greater than 0.3 ) satisfies the condition than the design is discarded.

\subsection{Calculating Magnetizing Current}

Material selected for the core is M-19 29 Gauge. Using the $\mathrm{BH}$ curve of the material and knowing the peak flux density $\left(B_{m}\right)$ in the yoke and limb, the MMF of the yoke $\left(M M F_{y}\right)$ and MMF of limb $\left(M M F_{l}\right)$ can be calculated. The magnetizing current required to saturate the transformer core to $B_{m}$ is then given as

$$
I_{m_{-} r m s}=\frac{M M F_{t o t}}{3 \cdot N 1 \cdot \sqrt{2}}=\frac{1.1 \cdot\left(M M F_{y}+M M F_{l}\right)}{3 \cdot N 1 \cdot \sqrt{2}}
$$

$10 \%$ increase in the value of MMF is considered in order to consider the flux concentration at the corners of the window.

\subsection{Calculating Power Loss}

With the defined geometric parameters, the resistance of the primary and secondary are computed in the conventional manner, and are used to determine the total copper losses $\left(P_{c u}\right)$.

$$
\begin{aligned}
& P_{c u}=P_{c u_{-} p}+P_{c u_{-} s}=3 \cdot R_{p} \cdot I_{p r m s}^{2}+3 \cdot R_{s} \cdot I_{s r m s}^{2} \\
& =3 \cdot\left(R_{p} \cdot I_{p r m s}^{2}+R_{s} \cdot I_{s r m s}^{2}\right)
\end{aligned}
$$

where, $R_{p}$ is primary winding phase resistance, $R_{s}$ is secondary winding phase, $I_{\text {prms }}$ is primary winding rms current and $I_{\text {srms }}$ is secondary winding rms current.

In the present work, the core losses are estimated using a modified Steinmetz equation given as

$$
P_{s 0}=\underbrace{k_{h} \cdot f^{\alpha} \cdot B_{m}{ }^{\beta}}_{\text {hysteresis }}+\underbrace{k_{e} \cdot\left(f \cdot B_{m}\right)^{2}}_{\text {eddy current }}
$$

where $k_{h}$ is coefficient for hysteresis losses, $k_{e}$ is the coefficient for the eddy current losses, $f$ is the fundamental frequency.

$P_{s 0}$ is core loss in $\mathrm{W} / \mathrm{kg}$ and hence, the total core loss $\left(P_{s}\right)$ is calculated using the weight of core (weight core $_{\text {e }}$ ) as

$$
P_{\text {core }}=P_{s 0} \cdot \text { weight }_{\text {core }}=P_{s 0} \cdot \rho_{s} \cdot \text { vol }_{\text {core }}
$$

where, $\rho_{s}$ is mass density of core material.

It is possible to calculate efficiency of the transformer using the power losses. The resulting efficiency must be higher than the expected design value. If this performance is not fulfilled, the design is discarded.

\subsection{Optimizing Stray Inductance}

In order to ensure soft switching of the $3 \mathrm{DAB}$ converter, a certain series inductance is required. Therefore, the selection of the stray inductance $\left(L_{\sigma}\right)$ has great impact on the performance of the $3 \mathrm{DAB}$ converter. The smaller $L_{\sigma}$ offers a wide range of soft-switching control for different operating voltages. $\varphi$ is decreased by decrease in $L_{\sigma}$ and hence, the turn off current on the switches is decreased resulting in minimizing the switching losses. The maximum value of $L_{\sigma}$ that ensures soft switching of $3 \mathrm{DAB}$ converter can be calculated as

$$
L_{\sigma}=\frac{V_{i n}^{2}}{\omega_{s w} P_{o}} \cdot d \cdot \phi\left[\frac{2}{3}-\frac{\phi}{2 \pi}\right] \quad \text { for }(0 \leq \phi \leq \pi / 3)
$$

$L_{\sigma}$ can be analytically calculated from the using geometric dimensions and is given as

$$
L_{\sigma}=\mu_{0} \cdot N 1^{2} \cdot \frac{M L T_{p}}{h} \cdot\left(\frac{w_{c u_{-} p}}{3}+d_{i n s_{-} p s}+\frac{w_{c u_{-} s}}{3}\right)
$$


where, $M L T_{P}$ is the mean length of the primary winding, $w_{c u \_p}$ is the width of the primary copper in the window, $d_{i n s_{-} p s}$ is the thickness of insulation between primary and secondary; and $w_{c u_{-} s}$ is the width of the secondary copper in the window.

The resulting transformer $L_{\sigma}$ must be smaller than or equal to the $3 \mathrm{DAB}$ converter $L_{\sigma}$. If the condition is not satisfied the design is discarded. For a 4MVA rated transformer for a $3 \mathrm{DAB}$ having $1.1 \mathrm{kVDC}$ input rating and 5.2kVDC input rating, the allowed maximum value of $L_{\sigma}$ is calculated as $25.2 \mu H$ and $563 \mu H$ respectively.

\subsection{Design Optimization}

In this design optimization, a set of five design variables is selected to be optimised for the transformer design. The design variables enlisted below.

1. $N_{l}$ is the number of turns of primary winding. The number of turns of secondary winding $\left(N_{2}\right)$ can be calculated using turns ratio.

2. $J_{p}$ is current density $\left[\mathrm{A} / \mathrm{m}^{2}\right]$ for primary windings.

3. $J_{s}$ is current density $\left[\mathrm{A} / \mathrm{m}^{2}\right]$ for secondary windings.

4. $w / h$ is the ratio of window width to window height.

5. $d / a$ is the ratio of window width to window height.

Window area ratio $w / h$ and core cross sectional area ratio $d / a$ are required to considered as design variables in order to optimise the transformer geometry.

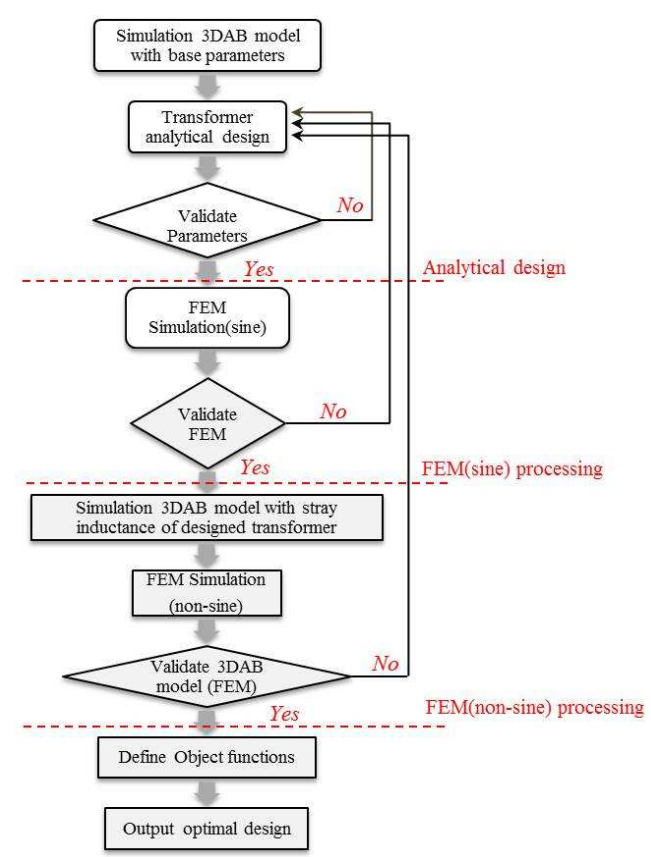

Fig. 5 Design Optimization Flowchart

Fig. 5 shows the flowchart showing the transformer design optimization algorithm. At first, the transformer specifications are determined from $3 \mathrm{DAB}$ converter in pre-design phase. A given set of design variable is selected and design of transformer is carried out as described in sections 3.1 to 3.5. Design that does not satisfy all the conditions specified in section 3.1 to 3.5 is discarded. The accepted design is validated using $2 \mathrm{D}$ transient FEA for sine excitation. 3DAB converter simulation is carried out using the stray inductance of the designed transformer and actual non-sine excitation waveforms are generated. Using this non-sine excitation waveform, again a 2D transient FEA is performed to compute the losses for non-sine excitation. Finally the efficiency is calculated and if it is greater the desired value the design is kept or else discarded. This procedure is repeated by varying the design variables until the full design space is spanned. Once, the design space is spanned completely, the generated designs are arranged according to the value of objective function and an optimal design is selected.

\subsection{Objective Function}

Most important parameters of designed transformer for 3DAB are efficiency, magnetizing current, copper losses, core losses and total weight. Efficiency is required to be maximized while magnetizing current, copper losses, core losses and total weight are required to be minimized. So, an objective function that satisfies this condition is selected for the optimization procedure and is given by Equation [19].

$$
\begin{gathered}
\eta_{\max }=\min \left(P_{\text {core }}, P_{c u}, I_{\text {mag }}, W_{\text {tot }}\right) \text { and } \geq \eta_{\mathrm{e}} \\
o b j_{\text {fun }}=\frac{\eta_{\mathrm{e}}}{P_{\text {core }} \cdot P_{c u} \cdot I_{m a g} \cdot W_{\text {tot }}}
\end{gathered}
$$

\section{Optimized Transformer Designs}

Optimal design of transformer is generated for $1.1 \mathrm{kVDC}$ and $5.2 \mathrm{kVDC}$ rated input voltage using the optimization algorithm discussed in section 3.6. Fig. 6 shows the variation of the efficiency with respect to total weight and stray inductance for both the $1.1 \mathrm{kVDC}$ and $5.2 \mathrm{kVDC}$ rated input voltage. Pink squares show the selected designs for each voltage rating.

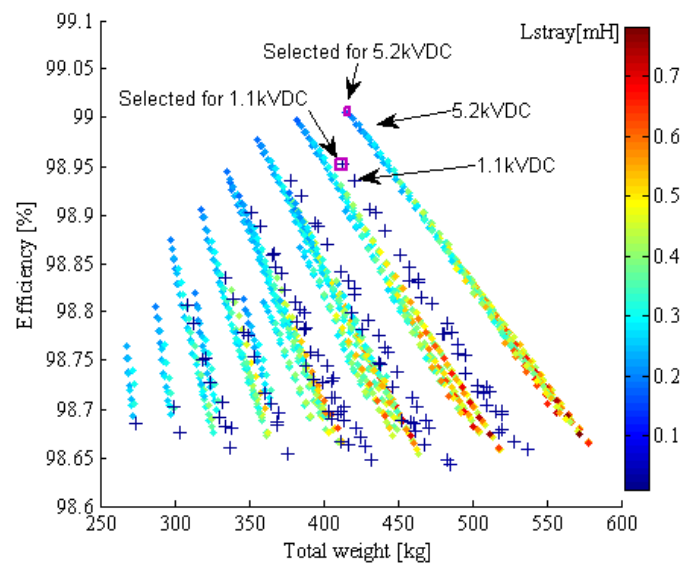

Fig. 6 Plot of various design scenarios 
Design details of these transformers, for $1.1 \mathrm{kVDC}$ and $5.2 \mathrm{kVDC}$, are given in Table 1 .

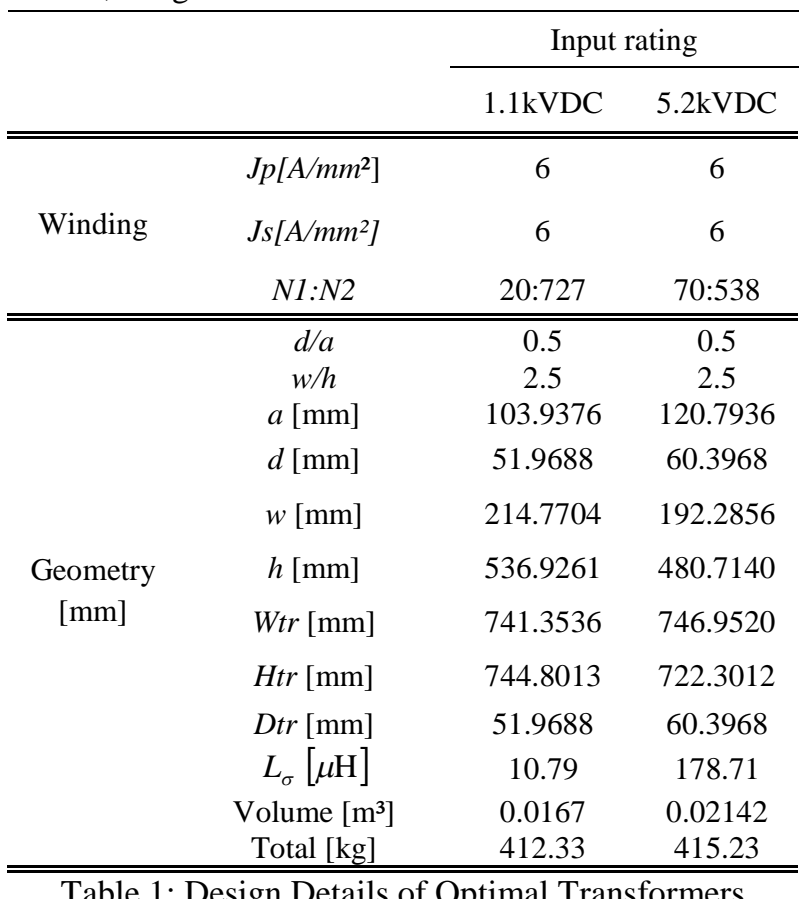

Table 1: Design Details of Optimal Transformers

The designed transformers are validated using 2D transient FEA. Table 2 shows the comparison between the results obtained analytically and obtained by 2D transient FEA.

\begin{tabular}{|c|c|c|c|c|}
\hline & & & \multicolumn{2}{|c|}{ Input rating } \\
\hline & & & $1.1 \mathrm{kVDC}$ & $5.2 \mathrm{kVDC}$ \\
\hline \multirow{8}{*}{ Losses } & \multirow{2}{*}{ Core $[\mathrm{kW}]$} & Analytical & 12.85 & 16.53 \\
\hline & & FEM & 11.81 & 14.99 \\
\hline & \multirow{2}{*}{$\begin{array}{c}\text { Winding } \\
{[\mathrm{kW}]}\end{array}$} & Analytical & 27.30 & 21.66 \\
\hline & & FEM & 27.24 & 21.69 \\
\hline & \multirow{2}{*}{$\operatorname{Imag}[\mathrm{A}]$} & Analytical & 226.54 & 60.17 \\
\hline & & FEM & 234.73 & 46.34 \\
\hline & \multirow{2}{*}{$\eta[\%]$} & Analytical & 98.90 & 98.92 \\
\hline & & FEM & 98.95 & 99.01 \\
\hline \multirow{2}{*}{\multicolumn{2}{|c|}{$\mathrm{Bm}[\mathrm{T}]$}} & Assumed & $\begin{array}{l}1.2 \\
1.870\end{array}$ & $\begin{array}{l}1.2 \\
1.565\end{array}$ \\
\hline & & FEM & 1.1829 & 1.1565 \\
\hline
\end{tabular}

Table 2: Performance Comparison of Optimal Transformers

The performance comparison presented here shows a good match between the analytical and the FEA.

The stray inductances of the optimized transformer are used for the $3 \mathrm{DAB}$ converter simulation. The resulting waveforms are shown in the Fig. 7 for both 1.1kVDC and 5.2kVDC rated input voltages.

The non-sinusoidal excitation waveforms, generated from $3 \mathrm{DAB}$ converter simulation, are then used to perform a $2 \mathrm{D}$ transient FEA to determine the performance of the designed transformer for the actual (non-sinusoidal) excitation. The plot of time average total losses of the transformer designed for $1.1 \mathrm{kVDC}$ and $5.2 \mathrm{kVDC}$ under sine wave excitation and non-sine wave excitation are shown in Fig. 8 and Fig. 9 respectively.
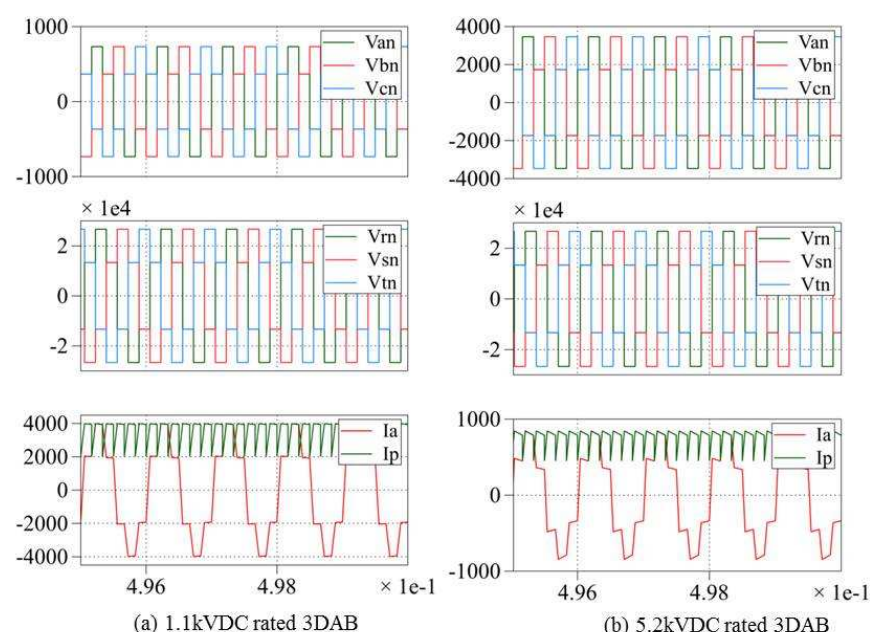

Fig.7. 3DAB simulation for the optimized transformer (a) $1.1 \mathrm{kVDC}$ rated voltage and (b) $5.2 \mathrm{kVDC}$ rated voltage

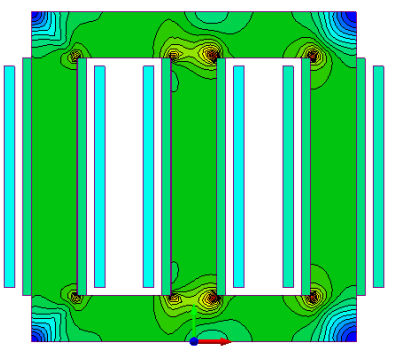

(a)

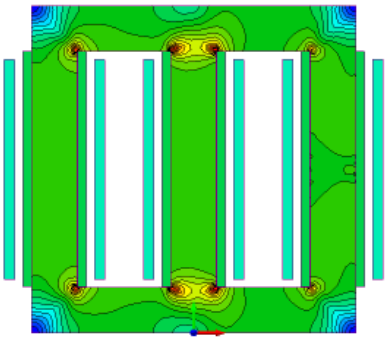

(b)
Fig. 8 Average total losses of (a) sinusoidal and (b) nonsinusoidal excitations for a $1.1 \mathrm{kVDC}$ rated input voltage

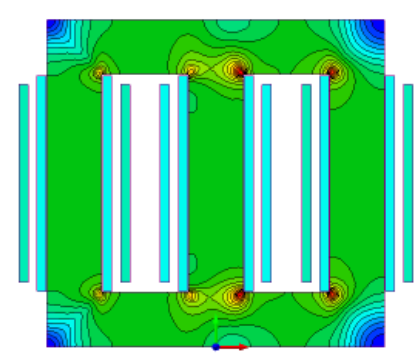

(a)

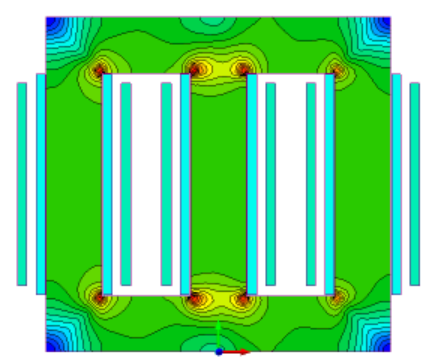

(b)
Fig. 9 Average total losses of (a) sinusoidal and (b) nonsinusoidal excitations for a 5.2kVDC rated input voltage

The comparison of losses for the sinusoidal and nonsinusoidal excitation for the optimized transformer designed for $1.1 \mathrm{kVDC}$ and $5.2 \mathrm{kVDC}$ rated input voltage are shown in Table 3 and Table 4 respectively.

\begin{tabular}{cccc}
\hline \multicolumn{2}{c}{ 4MVA, N1=20 } & Non-sinusoidal & Sinusoidal \\
\hline \multicolumn{2}{c}{ Core loss $[\mathrm{kW}]$} & 13.159 & 11.811 \\
$\mathrm{Cu}$ loss & Primary & 9.376 & 7.813 \\
{$[\mathrm{~kW}]$} & Secondary & 17.861 & 14.887 \\
\hline
\end{tabular}

Table 3: Comparison of losses between sinusoidal and nonsinusoidal for $1.1 \mathrm{kVDC}$ rated input voltage 


\begin{tabular}{cccc}
\hline \multicolumn{2}{c}{ 4MVA, N1=70 } & Non-sinusoidal & Sinusoidal \\
\hline \multicolumn{2}{c}{ Core loss $[\mathrm{kW}]$} & 16.654 & 14.993 \\
$\mathrm{Cu}$ loss & Primary & 6.982 & 5.818 \\
{$[\mathrm{~kW}]$} & Secondary & 14.706 & 12.207 \\
\hline
\end{tabular}

Table 4: Comparison of losses between sinusoidal and nonsinusoidal for $5.2 \mathrm{kVDC}$ rated input voltage

\section{Conclusions}

Isolation transformer is a key component of $3 \mathrm{DAB}$ DC-DC converter which is again an important part of DC-DC conversion (DC grid). Transformer is used for not only providing the galvanic isolation but also to change the voltage levels i.e. step up or step down (essential for power transmission). The work presented deals with the design and optimization of the transformer for 3DAB converter under the actual (non-sine) excitation for a 4MVA transformer of $3 \mathrm{DAB}$ converter, having $1.1 \mathrm{kV}$ and $5.2 \mathrm{kVDC}$ rated input voltages respectively. The results obtained from the analytical design are compared with the 2D transient FEA and shows a good match. The analytical results are always overestimating and hence, the final design would always perform better than the expectation. Targeted efficiency was $98.5 \%$ and the designed transformers for the $1.1 \mathrm{kVDC}$ and $5.2 \mathrm{kVDC}$ were able to achieve $98.95 \%$ and $99.01 \%$ efficiency respectively.

There is increase in the core losses, from sinusoidal to nonsinusoidal excitation, by approximately $15 \%$, and hence, it is essential to carry out the performance analysis for the actual $3 \mathrm{DAB}$ converter waveforms. The design optimization takes in to the account the non-sinusoidal excitation generated by simulation inside the optimization algorithm for the performance calculation of the designed transformer.

\section{Nomenclature}

$V_{a n} \quad$ Phase voltage on primary side

$f_{s w} \quad$ Switching frequency

$\varphi \quad$ Phase shift angle

$\omega_{s w} \quad$ Switching frequency

[rad]

$a \quad$ Width of core sectional area

$d \quad$ Length of core sectional area

$\eta_{e} \quad$ Estimated efficiency

$I_{m a g} \quad$ Magnetizing Current

$[\mathrm{rad} / \mathrm{s}]$

[mm]

[mm]

$W_{t o t} \quad$ Total weight of transformer

[kg]

\section{References}

[1] D. Jovcic and N. Strachan, "Offshore Wind Farm with Centralised Power Conversion and DC Interconnection," IET Generation Transmission \& Distribution, volume 3, pp. 586-595, (2009).
[2] D. V. H. D. Jovcic, K. Linden, J.-P. Taisne, and G. Wolfgang, "Feasibility of DC Transmission Networks," 2nd IEEE PES International Conference and Exhibition, pp. 1-8, (2011).

[3] [Online]. Available: http://new.abb.com/motorsgenerators

[4] [Online]. Available: http://www.ge-energy.com/

[5] R. Dedoncker, D. M. Divan, and M. H. Kheraluwala, "A 3-Phase Soft-Switched High-Power-Density DC-DC Converter for High-Power Applications," IEEE Transactions on Industry Applications, volume 27, pp. 63-73, (1991).

[6] N. Soltau, D. Eggers, K. Hameyer, and R. W. De Doncker, "Iron Losses in a Medium-Frequency Transformer Operated in a High-Power DC-DC Converter," IEEE Transactions on Magnetics, volume $50,(2014)$

[7] A. J. Moses and B. Thomas, "Problems in Design of Power Transformers," IEEE Transactions on Magnetics, volume 10, pp. 148-150, (1974).

[8] M. G. Say, Alternating Current Machines, Fifth Edition ed.: Longman Scientific \& Technical, (1983).

[9] J. W. Nims, R. E. Smith, and A. A. ElKeib, "Application of a genetic algorithm to power transformer design," Electric Machines and Power Systems, volume 24, pp. 669-680, (1996).

[10] P. S. Georgilakis, "Transformer Design Optimization," Spotlight on Modern Transformer Design, pp. 331-376, (2009).

[11] G. Ortiz, J. Biela, and J. W. Kolar, "Optimized Design of Medium Frequency Transformers with High Isolation Requirements," 36th Annual Conference of the IEEE Industrial Electronics Society, pp. 631-638, (2010).

[12] D. Vinnikov, J. Laugis, and I. Galkin, "MiddleFrequency Isolation Transformer Design Issues for the High-Voltage DC/DC Converter," IEEE Power Electronics Specialists Conference, pp. 1930-1936, (2008).

[13] S. Meier, T. Kjellqvist, S. Norrga, and H.-P. Nee, "Design Considerations for Medium-Frequency Power Transformers in Offshore Wind Farms," 13th European Conference on Power Electronics and Applications, pp.1-12, (2009).

[14] K. D. Hoang and J. Wang "Design Optimization of High Frequency Transformer for Dual Active Bridge DC-DC Converter," Xxth International Conference on Electrical Machines (ICEM), pp. 2311-2317, (2012).

[15] J. Zhang, Y. Du, Z. Li, and P. Wang, "Design of a Medium Frequency, High Voltage Transformer for Power Electronic Transformer," IEEE Transportation Electrification Conference and Expo (ITEC) AsiaPacific, pp. 1-5, (2014).

[16] C. Meyer, "Key Components for Future Offshore DC Grids," PhD. dissertation, Institute for Power Electronics and Electrical Drives, RWTH Achen University, (2007). 